\title{
Time-dependent density-functional theory with optimized effective potential and self-interaction correction and derivative discontinuity for the treatment of double ionization of $\mathrm{He}$ and Be atoms in intense laser fields
}

\author{
John Heslar, ${ }^{1}$ Dmitry A. Telnov, ${ }^{2}$ and Shih-I Chu ${ }^{1,3, *}$ \\ ${ }^{1}$ Center for Quantum Science and Engineering, and Center for Advanced Study in Theoretical Sciences, Department of Physics, \\ National Taiwan University, Taipei 10617, Taiwan \\ ${ }^{2}$ Department of Physics, St. Petersburg State University, St. Petersburg 198504, Russia \\ ${ }^{3}$ Department of Chemistry, University of Kansas, Lawrence, Kansas 66045, USA
}

(Received 27 July 2012; revised manuscript received 14 January 2013; published 22 May 2013)

\begin{abstract}
We present a self-interaction-free time-dependent density-functional theory (TDDFT) for the treatment of double-ionization processes of many-electron systems. The method is based on the extension of the Krieger-LiIafrate (KLI) treatment of the optimized effective potential (OEP) theory and the incorporation of an explicit self-interaction correction (SIC) term. In the framework of the time-dependent density functional theory, we have performed three-dimensional (3D) calculations of double ionization of $\mathrm{He}$ and $\mathrm{Be}$ atoms by intense near-infrared laser fields. We make use of the exchange-correlation potential with the integer discontinuity which improves the description of the double-ionization process. We found that a proper description of the double ionization requires the TDDFT exchange-correlation potential with the discontinuity with respect to the variation of the total particle number (TPN). The results for the intensity-dependent rates of double ionization of $\mathrm{He}$ and Be atoms are presented.

DOI: 10.1103/PhysRevA.87.052513

PACS number(s): 31.15.ee, 32.80.Rm
\end{abstract}

\section{INTRODUCTION}

Recent experimental progress in the generation of ultrashort xuv laser pulses has made possible the exploration of complete-breakup problems of atoms and molecules on a smaller time scale. Using subfemtosecond (sub-fs) xuv pulses allows us to peer into the exciting processes related to atomic inner-shell spectroscopy. The complete photoninduced breakup problem of many-electron atoms presents to theorists great challenges not found in single-ionization processes. As a prototype to understand the mechanisms of the multiple ionization process in complicated atomic targets, we focus ourselves on the double photoionization of helium and beryllium atoms. Even for the simplest two-electron atomic system, nonperturbative treatment for the double ionization still presents considerable computational challenges. Different theoretical models have been suggested to describe nonsequential double ionization (NSDI) (see, for example, review [1]). However, further experimental investigations [2-4] unambiguously pointed out that the dominant contribution to NSDI in the tunneling-ionization regime can be explained by the recollision mechanism [5]. According to the classical rescattering model [5], an electron removed from its atom by an external field can return to the parent ion after some period of time. When it returns, its kinetic energy can be as large as $3.17 U_{p}$ because of acceleration by the external field $\left[U_{p}\right.$ is the ponderomotive potential; for linearly polarized laser fields, $U_{p}=F^{2} /(2 \omega)^{2}$ where $F$ is the peak electric-field strength and $\omega$ is the laser frequency]. The returning electron can undergo elastic or inelastic scattering or it can recombine with the core. The latter possibility leads to emission of a photon (high-order harmonic generation, $\mathrm{HHG}$ ), and elastic scattering contributes to above-threshold ionization (ATI). Inelastic scattering results

\footnotetext{
*sichu@ku.edu
}

in excitation of the core or ejection of another electron through the $(e, 2 e)$ process; removal of multiple electrons is also possible. Of course, these inelastic processes can take place only if they obey the energy conservation.

The experimentally confirmed $(e, 2 e)$ mechanism of NSDI in the tunneling regime implies that proper account of the electron-electron interaction is crucial for a correct description of this process. It is not surprising that single active electron (SAE) theoretical models are unable to reproduce NSDI characteristics even qualitatively since such models do not include multielectron dynamics. On the contrary, the time-dependent density functional theory (TDDFT), in principle, contains all multielectron effects through the exchange-correlation functional. However, early attempts to apply TDDFT with various known exchange-correlation functionals for calculations of NSDI $[6,7]$ were unsuccessful. The calculations failed to describe the experimental results for double ionization of $\mathrm{He}$ atoms even qualitatively: the famous "knee" structure in the intensity dependence of the double-ionization probability $[8,9]$ could not be reproduced. Nowadays it is understood that the problem is not in TDDFT itself but in the quality of the exchange-correlation functionals. Most of approximate exchange-correlation functionals lack the important property of the exact functional: the discontinuity of its derivative with respect to the number of particles $N$ when $N$ passes through integer values [10]. It was shown [11] recently that the derivative discontinuity is crucial for a correct description of double ionization: a simple model correlation potential with the discontinuity being used in the time-dependent Kohn-Sham equations produced the intensity dependence of the double-ionization probability with the knee structure, in qualitative agreement with experiment. In the ground-state density functional theory, the exchange-correlation potentials with the discontinuity can be constructed with the help of the optimized effective potential (OEP) method and its simplified version, the Krieger-Li-Iafrate (KLI) approximation [12]. Both 
OEP and KLI can be extended to the TDDFT domain $[13,14]$, and the constructed time-dependent exchange-correlation potential retains the discontinuity property. The proposed timedependent KLI self-interaction correction (SIC) procedure, similar to the original KLI method, allows also the construction of a self-interaction-free effective potential that is orbital independent [15]. Moreover, it was demonstrated [16] that the discontinuity of the exchange-correlation potential is explicitly built up during the time propagation of the timedependent Kohn-Sham orbitals. It should be noted that the KLI-SIC potential, like many other orbital density dependent quantities, is not invariant under unitary transformations of the Kohn-Sham orbitals. Additional constraints (see, e.g., Ref. [17]) can be applied to obtain an optimal set of orbitals that minimizes the SIC energy. Usually, the optimal set consists of spatially localized orbitals. Recently, a generalized formulation of time-dependent OEP and KLI methods was suggested [18] which employs two sets of orbitals related by a unitary transformation. However, while this issue appears quite important for extended systems such as molecules or clusters, it is less important for atoms where the orbitals are naturally localized. Since we focus on NSDI of atoms in this work, we will follow the conventional formulation of the time-dependent KLI-SIC (TD-KLI-SIC) procedure. We also note that invariance under unitary transformations of the orbitals does not ensure a correct description of double ionization as well as lack of this invariance does not prevent it. The derivative discontinuity property is much more important in this respect. For example, the time-dependent Hartree-Fock approach cannot reproduce the knee structure in double ionization of $\mathrm{He}$; although the functional is invariant under unitary transformations of the orbitals in this case, it does not possess the derivative discontinuity property.

In our previous study [19], we found that the discontinuity of the TD-KLI-SIC potential related to the spin particle number (SPN) is not sufficient to reproduce characteristic features of double ionization. In the present paper, we extend the TD-KLI-SIC method where the exchange-correlation potential changes discontinuously when the total particle number (TPN) passes through an integer value. We use this new method to study double ionization of $\mathrm{He}$ and $\mathrm{Be}$ atoms by strong near-infrared laser fields, and the famous knee structure is reproduced with the use of TD-KLI-SIC.

\section{THEORY AND COMPUTATIONAL DETAILS}

The single-particle potential with the discontinuity is constructed with the help of TD-KLI-SIC procedure. Within the adiabatic approximation, well justified in the case of lowfrequency laser fields [20], the TD-KLI-SIC single-particle potential can be expressed as follows:

$$
V_{\sigma}^{\mathrm{SIC}}(\boldsymbol{r}, t)=\sum_{i=1}^{N_{\sigma}} \frac{\rho_{i \sigma}(\boldsymbol{r}, t)}{\rho_{\sigma}(\boldsymbol{r}, t)}\left[v_{i \sigma}(\boldsymbol{r}, t)+\bar{V}_{i \sigma}^{\mathrm{SIC}}-\bar{v}_{i \sigma}\right] .
$$

Here indices $i$ and $\sigma$ enumerate spin orbitals ( $\sigma$ corresponds to the spin projection), and $N_{\sigma}$ is the SPN (the number of electrons with the spin $\sigma$; the TPN $N$ is the total number of electrons: $N=N_{\uparrow}+N_{\downarrow}$ ). The spin-orbital density $\rho_{i \sigma}$ and the total spin density $\rho_{\sigma}$ are defined a follows:

$$
\rho_{i \sigma}(\boldsymbol{r}, t)=\left|\psi_{i \sigma}(\boldsymbol{r}, t)\right|^{2}, \quad \rho_{\sigma}(\boldsymbol{r}, t)=\sum_{i=1}^{N_{\sigma}} \rho_{i \sigma}(\boldsymbol{r}, t),
$$

where $\psi_{i \sigma}(\boldsymbol{r}, t)$ is the Kohn-Sham spin orbital.

The orbital-dependent potential $v_{i \sigma}(\boldsymbol{r}, t)$ includes the Hartree and exchange-correlation parts as well as selfinteraction corrections. In Eq. (1), the last two terms $\bar{V}_{i \sigma}^{\mathrm{SIC}}$ and $\bar{v}_{i \sigma}$ are constants, though the value of $\bar{V}_{i \sigma}^{\mathrm{SIC}}$ is unknown. The KLI method suggests a way to calculate $\bar{V}_{i \sigma}^{\text {SIC }}-\bar{v}_{i \sigma}$ through a solution of linear equations [15]. Following the KLI-SIC procedure, one finds that

$$
\begin{aligned}
\bar{V}_{i \sigma}^{\mathrm{SIC}} & =\int d^{3} r \rho_{i \sigma}(\boldsymbol{r}, t) V_{\sigma}^{\mathrm{SIC}}(\boldsymbol{r}, t), \\
\bar{v}_{i \sigma} & =\int d^{3} r \rho_{i \sigma}(\boldsymbol{r}, t) v_{i \sigma}(\boldsymbol{r}, t) .
\end{aligned}
$$

Equation (1) defines the potential $V_{\sigma}^{\text {SIC }}$ up to an arbitrary constant. However, since the exchange-correlation potential vanishes at infinity in the space domain, its expectation value with the highest-occupied spin orbital $\psi_{m \sigma}(\boldsymbol{r}, t)$ must be equal to that of the orbital-dependent potential $v_{m \sigma}(\boldsymbol{r}, t)$ [12]:

$$
\bar{V}_{m \sigma}^{\text {SIC }}=\bar{v}_{m \sigma} .
$$

The constraint (4) makes the potential (1) unique, and all unknown constants $\bar{V}_{i \sigma}^{\text {SIC }}(i<m)$ can be obtained solving a set of linear equations [12]. It is the constant $\bar{V}_{i \sigma}^{\text {SIC }}-\bar{v}_{i \sigma}$ in Eq. (1) that ensures discontinuous behavior of the TD-KLI potential with respect to the spin particle number $N_{\sigma}$ [16].

For $\mathrm{He}$ and $\mathrm{Be}$ atoms, the procedure is particularly straightforward since $N_{\sigma}$ does not exceed two. Since He $\left(1 s^{2}\right)$ and $\mathrm{Be}\left(1 s^{2} 2 s^{2}\right)$ are closed-shell atoms, the TD-KLI-SIC potential is spin independent. The helium atom has only one electronic shell; because of constraint (4), the potential $V_{\sigma}^{\mathrm{SIC}}(\boldsymbol{r}, t)$ reduces to the orbital-dependent potential $v_{1 \sigma}(\boldsymbol{r}, t)$. For the ion $\mathrm{He}^{+}$, there is only one electron which has a Coulomb interaction with the nucleus, and the potential $V_{\sigma}^{\mathrm{SIC}}(\boldsymbol{r}, t)$ vanishes. For the Be atom, the TD-KLI-SIC potential can be explicitly written as follows:

$$
\begin{aligned}
V_{\sigma}^{\mathrm{SIC}}(\boldsymbol{r}, t) & \\
= & \frac{\rho_{1 \sigma}(\boldsymbol{r}, t)}{\rho_{\sigma}(\boldsymbol{r}, t)}\left\{v_{1 \sigma}(\boldsymbol{r}, t)+\left[\int d^{3} r \frac{\rho_{2 \sigma}(\boldsymbol{r}, t) \rho_{1 \sigma}(\boldsymbol{r}, t)}{\rho_{\sigma}(\boldsymbol{r}, t)}\right]^{-1}\right. \\
& \left.\times \int d^{3} r \frac{\rho_{2 \sigma}(\boldsymbol{r}, t) \rho_{1 \sigma}(\boldsymbol{r}, t)}{\rho_{\sigma}(\boldsymbol{r}, t)}\left[v_{2 \sigma}(\boldsymbol{r}, t)-v_{1 \sigma}(\boldsymbol{r}, t)\right]\right\} \\
& +\frac{\rho_{2 \sigma}(\boldsymbol{r}, t)}{\rho_{\sigma}(\boldsymbol{r}, t)} v_{2 \sigma}(\boldsymbol{r}, t)
\end{aligned}
$$

For the open-shell $\mathrm{Be}^{+}$ion (the electronic structure $1 s^{2} 2 s$ ) the TD-KLI-SIC potential is spin dependent; the spin-up potential is still given by Eq. (6) while the spin-down potential reduces to the orbital-dependent potential $v_{1 \downarrow}(\boldsymbol{r}, t)$ :

$$
V_{\downarrow}^{\mathrm{SIC}}(\boldsymbol{r}, t)=v_{1 \downarrow}(\boldsymbol{r}, t) .
$$

For the orbital-dependent potentials $v_{i \sigma}(\boldsymbol{r}, t)$, we use the local-spin-density (LSD) approximation for both exchange and correlation. Regarding the LSD correlation part, we utilize numerical subroutines for the Perdew-Burke-Ernzerhof (PBE) 
TABLE I. Comparison of absolute values of spin-orbital energies (in a.u.) of $\mathrm{He}$ and $\mathrm{Be}$ atoms.

\begin{tabular}{lccc}
\hline \hline Atom & Spin orbital & Present calculations & Ref. \\
\hline $\mathrm{He}$ & $1 s$ & 0.918 & $0.904^{\mathrm{a}}$ \\
$\mathrm{Be}$ & $1 s$ & 4.039 & \\
& $2 s$ & 0.308 & $0.309^{\mathrm{b}}$ \\
\hline \hline
\end{tabular}

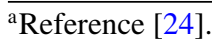

${ }^{\mathrm{b}}$ Reference [12].

functional [21] based on the analytical representation of the LSD correlation energy and potential by Perdew and Wang [22]. We also include Perdew-Zunger [23] self-interaction corrections:

$$
\begin{aligned}
v_{i \sigma}(\boldsymbol{r}, t)= & v_{\mathrm{H}}\left[\rho_{\uparrow}+\rho_{\downarrow}\right](\boldsymbol{r}, t)+v_{\mathrm{xc}}\left[\rho_{\sigma}\right](\boldsymbol{r}, t) \\
& -v_{\mathrm{H}}\left[\rho_{i \sigma}\right](\boldsymbol{r}, t)-v_{\mathrm{xc}}\left[\rho_{i \sigma}\right](\boldsymbol{r}, t),
\end{aligned}
$$

where $v_{\mathrm{H}}[\rho](\boldsymbol{r}, t)$ and $v_{\mathrm{xc}}[\rho](\boldsymbol{r}, t)$ are the Hartree and LSD exchange-correlation potentials, respectively:

$$
\begin{gathered}
v_{\mathrm{H}}[\rho](\boldsymbol{r}, t)=\int d^{3} r^{\prime} \frac{\rho\left(\boldsymbol{r}^{\prime}, t\right)}{\left|\boldsymbol{r}-\boldsymbol{r}^{\prime}\right|}, \\
v_{\mathrm{xc}}[\rho](\boldsymbol{r}, t)=-\left[\frac{6}{\pi} \rho(\boldsymbol{r}, t)\right]^{1 / 3}+v_{\mathrm{c}}^{\mathrm{LSD}}[\rho](\boldsymbol{r}, t) .
\end{gathered}
$$

The spin-orbital energies of the ground states computed by the time-independent DFT using these potentials are listed in Table I. They are in good agreement with the results of precision two-electron variational calculations of He [24] and accurate Hartree-Fock and OEP calculations of Be [12]. To obtain the time-dependent electron densities and calculate the ionization probabilities, one has to solve a set of the the timedependent Kohn-Sham equations for the spin orbitals $\psi_{i \sigma}(\boldsymbol{r}, t)$ :

$$
\begin{aligned}
& i \frac{\partial}{\partial t} \psi_{i \sigma}(\boldsymbol{r}, t) \\
& \quad=\left[-\frac{1}{2} \nabla^{2}-\frac{Z}{r}+V_{\sigma}^{\mathrm{SIC}}(\boldsymbol{r}, t)+v_{\mathrm{ext}}(\boldsymbol{r}, t)\right] \psi_{i \sigma}(\boldsymbol{r}, t), \\
& i=1, \ldots, N_{\sigma} .
\end{aligned}
$$

Besides the discussed single-particle potential $V_{\sigma}^{\text {SIC }}$, the right-hand side of Eq. (10) contains the Coulomb interaction with the nucleus ( $Z$ is the nucleus charge) and interaction with the external laser field $v_{\text {ext }}(\boldsymbol{r}, t)$. In our calculations we use a linearly polarized laser pulse:

$$
v_{\mathrm{ext}}(\boldsymbol{r}, t)=\boldsymbol{F}(t) \cdot \boldsymbol{r} .
$$

The wavelength of the carrier is $780 \mathrm{~nm}$ and $1200 \mathrm{~nm}$ for the $\mathrm{He}$ and Be atom, respectively. The total pulse duration $T$ is 60 optical cycles $(\sim 160$ fs $)$ for the helium atom and 30 optical cycles for the beryllium atom. The pulse envelope consists of a flat central part (50 optical cycles for He and 20 optical cycles for $\mathrm{Be}$ ) and five-optical-cycle sine-squared ramps at the leading and trailing edges:

$$
\begin{gathered}
\boldsymbol{F}(t)=\boldsymbol{F}_{0} \sin ^{2}(\Omega t) \sin \left(\omega_{0} t\right), \quad 0 \leqslant t<\tau, \\
\boldsymbol{F}(t)=\boldsymbol{F}_{0} \sin \left(\omega_{0} t\right), \quad \tau \leqslant t \leqslant T-\tau, \\
\boldsymbol{F}(t)=\boldsymbol{F}_{0} \sin ^{2}(\Omega t) \sin \left(\omega_{0} t\right), \quad T-\tau<t \leqslant T,
\end{gathered}
$$

where $\tau=10 \pi / \omega_{0}, \Omega=\omega_{0} / 20$.
To solve the set of equations (10), we apply the time-dependent generalized pseudospectral (TDGPS) method which proved accurate and efficient in our previous TDDFT calculations (see, e.g., Refs. [25-27]). For the TDGPS discretization in the present calculations, we use 256 radial and 32 angular grid points, and the time step 0.027 a.u. Equations (10) are solved in space within a sphere with the radius 60 a.u.; between 40 a.u. and 60 a.u. we place an absorber. Absorbed parts of the wave packet localized beyond 40 a.u. describe unbound states populated during the ionization process. Because of the absorber, the normalization integrals of the spin-orbital densities $\rho_{i \sigma}(\boldsymbol{r}, t)$ decrease in time. Calculated after the pulse, they determine the ionization probabilities $P_{i \sigma}$ for each spin orbital:

$$
P_{i \sigma}=1-\int d^{3} r \rho_{i \sigma}(\boldsymbol{r}, T) .
$$

We note that, for the peak intensities used in the calculations (up to $5 \times 10^{14} \mathrm{~W} / \mathrm{cm}^{2}$ ), only the highest-occupied $2 s$ orbital of $\mathrm{Be}$ is ionized while the tightly bound inner shell $1 s$ electrons do not leave the core. Also, the He atom is completely ionized (both electrons are removed) at $1 \times 10^{16} \mathrm{~W} / \mathrm{cm}^{2}$. Now we define the average single- and double-ionization rates. The concept of the ionization rate is based on the exponential decay law when the time-dependent ionization probability $\mathcal{P}_{\text {ion }}(t)$ can be represented as follows:

$$
\mathcal{P}_{\text {ion }}(t)=1-\exp (-\Gamma t) .
$$

In this case, the ionization rate $\Gamma$ is a constant and can be calculated at any time according to the equation

$$
\Gamma=-\frac{d}{d t} \ln \left[1-\mathcal{P}_{\text {ion }}(t)\right] .
$$

For laser pulses, when the intensity varies in time, the exponential decay law is not satisfied. However, we still can define the average ionization rate using the values of the pulse duration $T$ and ionization probability at the end of the pulse $\mathcal{P}_{\text {ion }}$ :

$$
\Gamma=\frac{1}{T} \ln \left(\frac{1}{1-\mathcal{P}_{\text {ion }}}\right) .
$$

Then for He and Be atoms, the single- and double-ionization rates $\Gamma^{(1)}$ and $\Gamma^{(2)}$ can be calculated as follows:

$$
\Gamma^{(1)}=\frac{1}{T} \ln \left(\frac{1}{1-\mathcal{P}^{(1)}}\right), \quad \Gamma^{(2)}=\frac{1}{T} \ln \left(\frac{1}{1-\mathcal{P}^{(2)}}\right),
$$

where the single- and double-ionization probabilities after the pulse, $\mathcal{P}^{(1)}$ and $\mathcal{P}^{(2)}$, are defined as

$$
\mathcal{P}^{(1)}=2 P_{m \sigma}\left(1-P_{m \sigma}\right), \quad \mathcal{P}^{(2)}=P_{m \sigma}^{2} .
$$

Here $m=1$ for $\mathrm{He}$ and $m=2$ for Be. The definitions of the single- and double-ionization probabilities in Eq. (20) have a clear physical meaning and are known as a meanfield approximation. They appear exact only for uncorrelated systems where the wave functions can be represented by Slater determinants [6]. Nevertheless, the approximation [Eq. (20)] is widely used and gives reasonable results for both single- and double-ionization probabilities if accurate electron densities 
are supplied for calculation of the spin-orbital ionization probabilities. For example, when calculated with the exact time-dependent electron density of $\mathrm{He}$, it reproduces the knee structure in the intensity dependence of the double-ionization probability [7]. Here we extend this approximation to calculate single- and double-ionization rates according to Eq. (19).

In TDDFT, for closed-shell atoms, the electrons with opposite spin projections are equally coupled to the laser field and can be detached from the core with equal probabilities. Then the single ionization (decrease of the total electron number $N=N_{\uparrow}+N_{\downarrow}$ by unity) corresponds to the decrease of each $N_{\uparrow}$ and $N_{\downarrow}$ by $1 / 2$, and the TD-KLI-SIC exchange potentials for both spins keep changing continuously. According to our modified TD-KLI-SIC scheme (the TPN approach), after single ionization $\left[\int d^{3} r \rho_{m \uparrow}(\boldsymbol{r}, t)=\int d^{3} r \rho_{m \downarrow}(\boldsymbol{r}, t)=0.5\right.$, with $m=1$ for $\mathrm{He}$ and $m=2$ for Be], the potentials in Eqs. (6) and (7) are changed discontinuously. For the spinup potentials, we replace the highest-occupied-shell spin-up density $\rho_{m \uparrow}(\boldsymbol{r}, t)$ with $2 \rho_{m \uparrow}(\boldsymbol{r}, t)$ and set $\rho_{m \downarrow}(\boldsymbol{r}, t)=0$. For the spin-down potentials, vice versa, the outer-shell spin-down density $\rho_{m \downarrow}(\boldsymbol{r}, t)$ is replaced with $2 \rho_{m \downarrow}(\boldsymbol{r}, t)$ and the spin-up density is set to zero. Thus the spin-up and spin-down potentials actually remain equal to each other and the system remains spin unpolarized. Upon the described transformation, however, from the physical point of view this system represents an equally populated statistical ensemble of open-shell ions with spin up and spin down. For example, for the He atom, the potential $V_{\sigma}^{\mathrm{SIC}}(\boldsymbol{r}, t)$ vanishes upon the transformation, as it supposed to be for the $\mathrm{He}^{+}$ion. We stress that the discontinuous transformation is applied to the potentials only. The Kohn-Sham spin orbitals $\psi_{i \sigma}(\boldsymbol{r}, t)$ in Eq. (10) keep changing continuously with time; so do the spin-orbital densities used to calculate the ionization probabilities and rates in Eqs. (15)-(20).

A sharp change of the potential occurs when the TPN rather than SPN passes through an integer number. Consequently, a TD-KLI-SIC exchange-correlation potential with the discontinuity with respect to the TPN variation is enforced. Otherwise, if we follow a conventional TD-KLI-SIC procedure (the SPN approach), the single ionization of the He $1 s$ orbital and the Be $2 s$ orbital corresponds to the condition $\int d^{3} r \rho_{m \downarrow}(\boldsymbol{r}, t)=$ $\int d^{3} r \rho_{m \uparrow}(\boldsymbol{r}, t)=0.5$, and there is no sharp change in the TDKLI-SIC exchange-correlation potential (which only occurs when the SPN passes through an integer number). In practice, when applying the TPN approach, we calculate the SPN at every time step during the time propagation of Kohn-Sham orbitals, and make the change of the exchange-correlation potential when the SPN drops by 0.5 . Our procedure allows the discontinuity in the TD-KLI-SIC exchange-correlation potential with respect to the TPN variation, and the doubleionization features can be reproduced. We emphasize that this is also true in the special case of the single-shell $\mathrm{He}$ atom where the potential (1) reduces to the form (7) with the LSD exchange-correlation (9) and does not exhibit a discontinuity with respect to the SPN. Although He does not represent a general case of the TD-KLI-SIC approach, it is a benchmark system for NSDI where the theory can be verified by the experiment. That is why we begin presentation of our numerical results with the data on the double ionization of He.

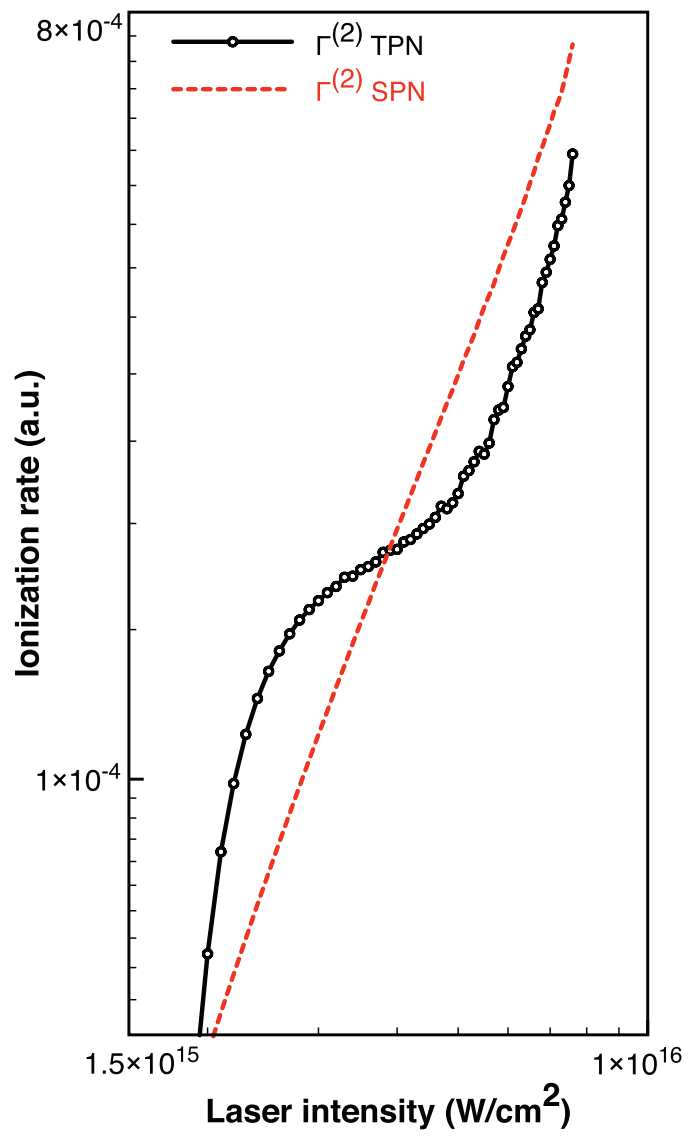

FIG. 1. (Color online) Double ionization rates of He atom versus the peak intensity of the laser pulse. The carrier wavelength is $780 \mathrm{~nm}$ and the pulse duration is 60 optical cycles.

\section{RESULTS AND DISCUSSION}

In Fig. 1, we compare the intensity dependence of the double-ionization rates of He calculated according to Eq. (19). We have obtained the ionization data with both the SPN and TPN versions of the TD-KLI-SIC exchange-correlation potential in the range of the peak intensities $1.5 \times 10^{15} \mathrm{~W} / \mathrm{cm}^{2}$ to $1 \times 10^{16} \mathrm{~W} / \mathrm{cm}^{2}$. While the results of the calculations resemble each other closely before the saturation of single ionization, thereafter, the TPN data for the double-ionization probability clearly exhibit the knee structure while the SPN curve does not have any similar features. The knee structure in Fig. 1 appears approximately at the same intensity as seen in the experiments of Refs. [8,9]. Thus we may conclude that the proper description of the double ionization requires the TDDFT exchange-correlation potential with the discontinuity with respect to the variation of the total particle numbers.

To illustrate the difference between the SPN and TPN approaches, it is instructive to compare the TD-KLI-SIC exchange-correlation potentials before and after the interaction with the laser pulse. The orbital-independent exchangecorrelation potential $V_{\mathrm{xc}, \sigma}(\boldsymbol{r}, t)$ is naturally defined as the difference between the single-particle potential $V_{\sigma}^{\mathrm{SIC}}(\boldsymbol{r}, t)$ and the Hartree potential $v_{\mathrm{H}}[\rho](\boldsymbol{r}, t)$ :

$$
V_{\mathrm{xc}, \sigma}(\boldsymbol{r}, t)=V_{\sigma}^{\mathrm{SIC}}(\boldsymbol{r}, t)-v_{\mathrm{H}}[\rho](\boldsymbol{r}, t) .
$$




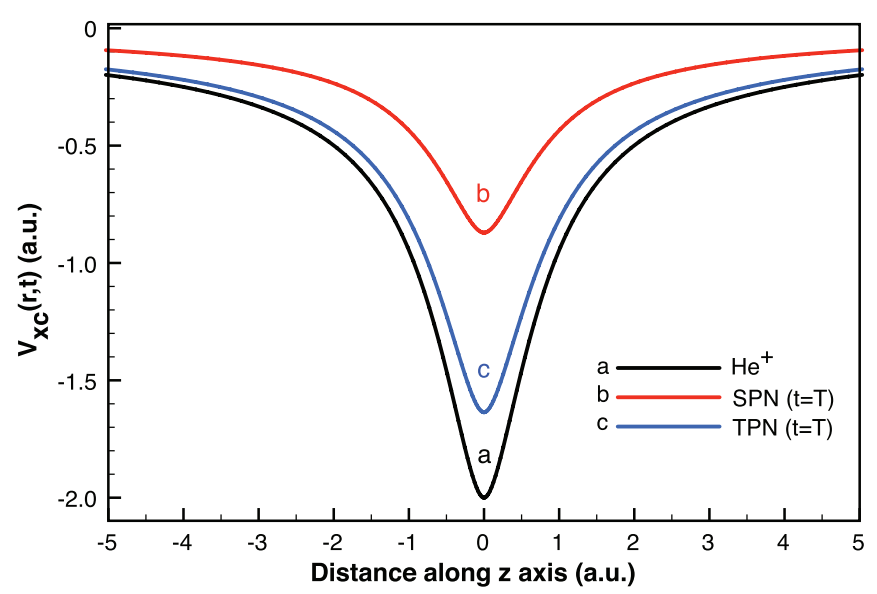

FIG. 2. (Color online) TD-KLI-SIC exchange-correlation potential $V_{\mathrm{xc}, \sigma}(\boldsymbol{r}, t)$ of the He atom at the end of the pulse $(t=T)$ for (b) SPN (red curve) and (c) TPN (blue curve) approaches. The peak intensity is $2.1 \times 10^{15} \mathrm{~W} / \mathrm{cm}^{2}$, the carrier wavelength is $780 \mathrm{~nm}$, and the pulse duration is 60 optical cycles. (a) The black curve shows the exchange-correlation potential $V_{\mathrm{xc}, \uparrow}(\boldsymbol{r})$ of the $\mathrm{He}^{+}$ion.

Defined in this way, the exchange-correlation potential includes the self-interaction correction and decays at large distances as $-1 / r$. In Fig. 2, we show the comparison between the SPN and TPN versions of the exchange-correlation potential for the He atom along the $z$ axis which coincides with the polarization direction of the laser field. The peak intensity of the laser is $2.1 \times 10^{15} \mathrm{~W} / \mathrm{cm}^{2}$ for both the SPN and TPN approaches. At this intensity, at the end of the pulse the He atom is singly ionized with high probability, and the single-particle potential $V_{\sigma}^{\mathrm{SIC}}(\boldsymbol{r}, T)$ is supposed to vanish. Hence the exchange-correlation potential must be equal to the negative Hartree potential for the $\mathrm{He}^{+}$ion. As one can see, the TPN approach reproduces this potential much better the SPN calculation. The existing difference between the TPN and $\mathrm{He}^{+}$exchange-correlation potentials can be explained by substantial double ionization at this intensity which affects the TPN potential through the density.

For the Be atom, we have obtained the double-ionization rates for the laser wavelength of $1200 \mathrm{~nm}$ with 30-optical-cycle pulses in the range of peak intensities $4.5 \times 10^{13} \mathrm{~W} / \mathrm{cm}^{2}$ to $5 \times 10^{14} \mathrm{~W} / \mathrm{cm}^{2}$, using both the SPN and TPN versions of the TD-KLI-SIC procedure. Figure 3 shows the double-ionization rates calculated according to Eq. (19) with high resolution in laser intensity. As one can see, the results for the TPN approach clearly reproduce the knee structure in the intensity dependence of the double-ionization probability.

In Fig. 4 we show the comparison between the SPN and TPN exchange-correlation potentials for the $\mathrm{Be}$ atom. Here, we choose the peak intensity $6 \times 10^{13} \mathrm{~W} / \mathrm{cm}^{2}$. At this intensity, at the end of the pulse the Be atom is singly ionized with high probability. The exchange-correlation potential for the TPN approach changes discontinuously in the course of interaction with the laser field when one of the $2 s$ electrons is detached; at the end of the laser pulse, it reproduces the exchange-correlation potential potential of $\mathrm{Be}^{+}$ion almost exactly. On the contrary, the exchange-correlation potential for the SPN approach has a continuous transformation during the interaction with the laser field. At the end of the pulse, it

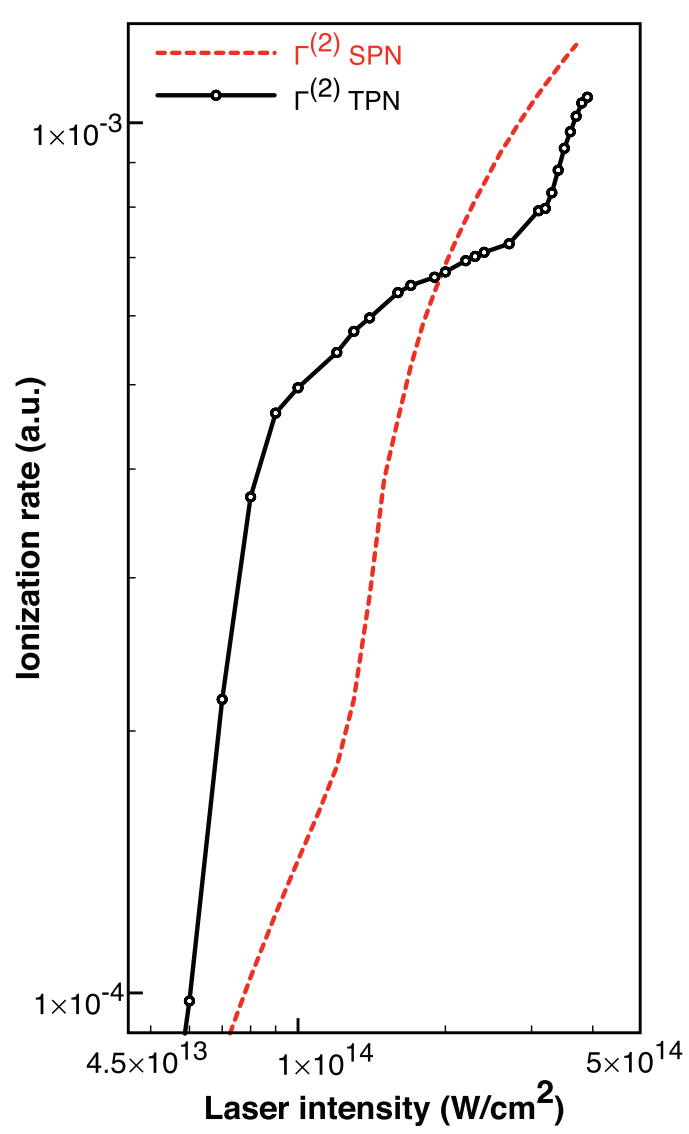

FIG. 3. (Color online) Double ionization rates of Be atom versus the peak intensity of the laser pulse. The carrier wavelength is $1200 \mathrm{~nm}$ and the pulse duration is 30 optical cycles.

remains close to the initial (neutral $\mathrm{Be}$ ) exchange-correlation potential at smaller distances corresponding to localization of

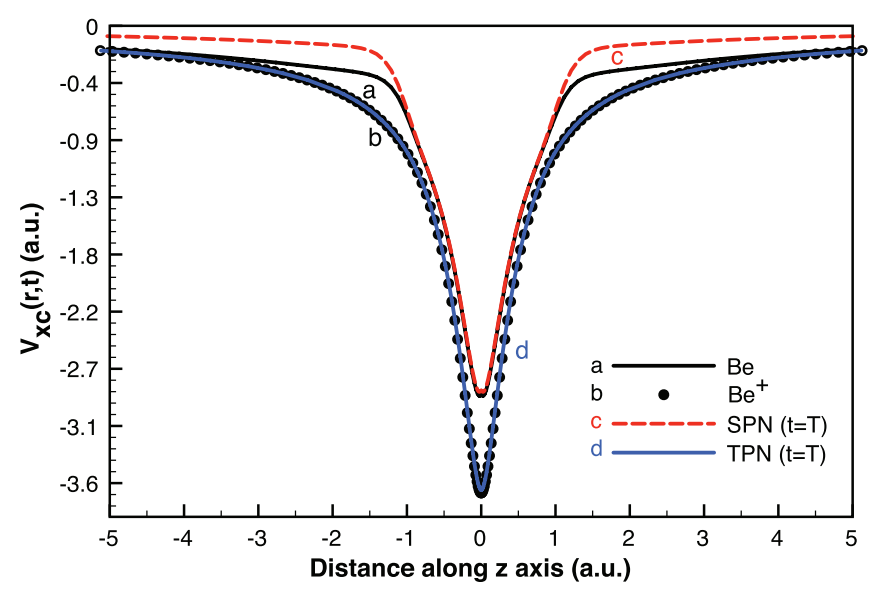

FIG. 4. (Color online) TD-KLI-SIC exchange-correlation potential $V_{\mathrm{xc}, \sigma}(\boldsymbol{r}, t)$ of Be atom, shown along polarization axis of the laser field (a) before the pulse ( $t=0$, black solid line). (c) SPN exchangecorrelation potential after the pulse ( $t=T$, red dashed line). (d) TPN exchange-correlation potential after the pulse $(t=T$, blue solid line). Black filled circles (b) correspond to the time-independent KLI-SIC exchange-correlation potential $V_{\mathrm{xc}, \downarrow}(\boldsymbol{r})$ of the $\mathrm{Be}^{+}$ion. The peak intensity of the laser field is $6 \times 10^{13} \mathrm{~W} / \mathrm{cm}^{2}$; the carrier frequency and pulse duration are the same as in Fig. 3. 
the electron density of $1 s$ electrons which are not affected much by the laser field; at larger distances, $|z| \gtrsim 1$ a.u., its deviation from the initial exchange-correlation potential accounts for the density change of the valence $2 s$ electrons due to the interaction with the laser field. However, the SPN exchange-correlation potential still differs significantly from that of $\mathrm{Be}^{+}$. This difference seen in Fig. 4 suggests that the SPN approach may not provide an adequate description of double ionization.

\section{SUMMARY}

We have performed 3D calculations of single and double ionization of $\mathrm{He}$ and $\mathrm{Be}$ atoms by using the self-interactionfree TDDFT approach. We have introduced the TD-KLISIC procedure for the exchange-correlation potential which contains a discontinuity with respect to the variation of the total particle numbers. Our exchange-correlation potential is nonadiabatic in the sense that it does not follow adiabatically the variation of the density. Instead, it undergoes a discontinuous transformation at some point that marks the single ionization (when the total particle number passes through an integer value). The discontinuity of the exchangecorrelation potential is explicitly manifested in the process of interaction with the laser field. We have presented the intensity-dependent rates for the double ionization of $\mathrm{He}$ and Be atoms reproducing the characteristics of the knee structure (which was experimentally observed in double ionization of He atoms). Thus we have shown that TDDFT employing the exchange-correlation potentials with the discontinuity with respect to the total particle number is capable of reproducing the characteristic features of double ionization.

\section{ACKNOWLEDGMENTS}

This work was partially supported by the US Department of Energy. We would like also to acknowledge the partial support of the National Science Council of Taiwan and National Taiwan University (Grants No. 101R8700-2 and No. 101R104021). D.A.T. acknowledges the partial support of St. Petersburg State University (Grant No. 11.38.654.2013).
[1] W. Becker and H. Rottke, Contemp. Phys. 49, 199 (2008).

[2] R. Moshammer, B. Feuerstein, W. Schmitt, A. Dorn, C. D. Schröter, J. Ullrich, H. Rottke, C. Trump, M. Wittmann, G. Korn, K. Hoffmann, and W. Sandner, Phys. Rev. Lett. 84, 447 (2000).

[3] A. Staudte, C. Ruiz, M. Schöffler, S. Schössler, D. Zeidler, Th. Weber, M. Meckel, D. M. Villeneuve, P. B. Corkum, A. Becker, and R. Dörner, Phys. Rev. Lett. 99, 263002 (2007).

[4] A. Rudenko, V. L. B. de Jesus, Th. Ergler, K. Zrost, B. Feuerstein, C. D. Schröter, R. Moshammer, and J. Ullrich, Phys. Rev. Lett. 99, 263003 (2007).

[5] P. B. Corkum, Phys. Rev. Lett. 71, 1994 (1993).

[6] M. Petersilka and E. K. U. Gross, Laser Phys. 9, 105 (1999).

[7] D. G. Lappas and R. van Leeuwen, J. Phys. B 31, L249 (1998).

[8] D. N. Fittinghoff, P. R. Bolton, B. Chang, and K. C. Kulander, Phys. Rev. Lett. 69, 2642 (1992).

[9] B. Walker, B. Sheehy, L. F. DiMauro, P. Agostini, K. J. Schafer, and K. C. Kulander, Phys. Rev. Lett. 73, 1227 (1994).

[10] J. P. Perdew, R. G. Parr, M. Levy, and J. L. Balduz, Jr., Phys. Rev. Lett. 49, 1691 (1982).

[11] M. Lein and S. Kümmel, Phys. Rev. Lett. 94, 143003 (2005).

[12] J. B. Krieger, Y. Li, and G. J. Iafrate, Phys. Rev. A 45, 101 (1992).
[13] C. A. Ullrich, U. J. Gossmann, and E. K. U. Gross, Phys. Rev. Lett. 74, 872 (1995).

[14] X. M. Tong and Shih-I Chu, Phys. Rev. A 57, 452 (1998).

[15] X. M. Tong and Shih-I Chu, Phys. Rev. A 55, 3406 (1997).

[16] M. Mundt and S. Kümmel, Phys. Rev. Lett. 95, 203004 (2005).

[17] M. R. Pederson, R. A. Heaton, and C. C. Lin, J. Chem. Phys. 80, 1972 (1984).

[18] J. Messud, P. M. Dinh, P.-G. Reinhard, and E. Suraud, Phys. Rev. Lett. 101, 096404 (2008).

[19] D. A. Telnov, J. Heslar, and S. I. Chu, Chem. Phys. 391, 88 (2011).

[20] M. Thiele, E. K. U. Gross, and S. Kümmel, Phys. Rev. Lett. 100, 153004 (2008).

[21] J. P. Perdew, K. Burke, and M. Ernzerhof, Phys. Rev. Lett. 77, 3865 (1996).

[22] J. P. Perdew and Y. Wang, Phys. Rev. B 45, 13244 (1992).

[23] J. P. Perdew and A. Zunger, Phys. Rev. B 23, 5048 (1981).

[24] Atomic, Molecular, and Optical Physics Handbook, edited by G. W. F. Drake (AIP, New York, 1996).

[25] D. A. Telnov and Shih-I Chu, Phys. Rev. A 80, 043412 (2009).

[26] J. Heslar, J. Carrera, D. Telnov, and S. I. Chu, Int. J. Quant. Chem. 107, 3159 (2007).

[27] X. M. Tong and S. I. Chu, Chem. Phys. 217, 119 (1997). 